\title{
Exercise - induced Anaphylaxis
}

Dr. Hima Hoskote, Dr. Adrian E Lopez Pradere

Luton and Dunstable University Hospital NHS Foundation Trust

\section{Background}

Exercise-induced anaphylaxis (EIA) is a rare disorder with a few well documented cases $(1,2)$, often associated with food allergy. Anaphylaxis itself has a high incidence of cross reactivity with many drugs used in Medicine in general, and in Anaesthetics, in particular. We present and highlight the anaesthetic management of an Obstetric case with this rare disorder who had two uneventful and successful pregnancies. On both occasions the patient was delivered at our hospital.

\section{Case Report}

A 36-year-old lady was seen at the antenatal clinic at 36 weeks, referred to us by her allergist. Three years earlier, she had noticed rashes and hives on her arm while dancing and the same episode occurred after a meal which resolved on topical application of ice. Over the following years, she reported further episodes of rashes, itching on her back and retro-auricular regions, more often during Zumba classes or following intense physical activity at work.Some of these episodes resulted in frank anaphylaxis with tongue swelling and respiratory distress, requiring hospital admissions to manage the anaphylaxis.

She tested negative to many common allergen food products at the allergy clinic. However, the occurrence of two of the anaphylactic episodes during or immediately after exercising and after a meal, suggested the diagnosis of a food-dependent, exercise induced anaphylaxis (FDEIA). She was advised to avoid wheat and intensive physical activities and prescribed an EPI pen for use as essential. With this regime, she has been free of such anaphylactic reactions to exercise and has had two successful pregnancies.

After the initial consultation in the antenatal clinic in 2013 it was suggested by the Anaesthetists to avoid going into labour, which was approved at the MDT. She was scheduled for an elective Caesarean section to avoid the stress of labour. Anti-histamine receptor antagonists including $4 \mathrm{mg}$ of chlorphenamine PO and $50 \mathrm{mg}$ of ranitidine PO along with $200 \mathrm{mg}$ of intravenous hydrocortisone as pre-medication were administered pre-operatively and a combined spinal epidural (CSE) technique was performed avoiding the use of intrathecal opioids. A similar approach was used again for her second Caesarean Section in 2016.

\section{Discussion}

Allergic reactions induced by physical activity, namely, EIA, is a rare and potentially serious condition that can pose challenges during stressful situations including pregnancy and labour.

Sheffer and Austen(1), in their series of sixteen cases, described four phases of anaphylaxis:

- prodromal phase with symptoms of fatigue, warmth, pruritus

- early phase with urticaria

- fully established stage with respiratory stridor, GI colic, nausea and choking

- late phase with frontal headaches that persisted for 24-72 hours

The type and degree of exercise to trigger this event have varied greatly, the most common being jogging (3). Many food products, most commonly wheat, have been associated with EIA. Other co-factors like alcohol, temperature, humidity, medications and hormonal changes have also been implicated for the precipitation of attacks(3).

The pathophysiological processes may include

- low threshold for mast cell degranulation

- histamine release

- disruption between pro-inflammatory and anti-inflammatory responses

- abnormalities of the autonomic nervous system, when associated with food allergy $(3,5)$.

The condition may be sporadic or familial. With no definitive cure, patients are managed by avoidance of trigger factors and symptomatic therapy (3). Omalizumab, an immunoglobulin $\mathrm{E}(\mathrm{IgE})$ monoclonal antibody that has been used to treat allergic asthma, has been reported to be successful in preventing and treating $\operatorname{EIA}(4,5)$.

Pregnancy induces wide-spread haemodynamic changes and physiological adaptations.During labour and delivery, the physiological changes mimic those observed during exercise. Together with dietary and hormonal changes, these could act as a potential trigger for anaphylaxis during pregnancy in this particular population. Both surgical and anaesthetic stress would compound the likelihood of any such precipitation More recently, Chaudry and McKay(6) described a similar case in Obstetrics managed successfully with regular intravenous hydrocortisone and chlorphenamine once labour was established with an early epidural for a normal assisted vaginal delivery.

However, in the present case, both pregnancies were successfully managed with a planned elective Caesarean section (CS) with a preanaesthetic work-up to avoid the stress of labour and a potential trigger of EIA. Administration of anti-histamine receptor antagonists like chlorphenamine (anti $\mathrm{H}-1$ ) and ranitidine ( anti $\mathrm{H}-2$ ) along with hydrocortisone as pre-medication were used to reduce the chance of mast cell degranulation. The avoidance of intrathecal opioids for the regional anaesthesia for CS was implemented to reduce the chances of histamine release. This approach ensured a safe and successful management of this complex obstetric case on both occasions.

\section{Learning Points}

1. Awareness about EIA.

2. A pre-determined anaesthetic management plan for patients with known generalised allergic reactions to non-specific allergens would lead to a comfortable, stress-free and successful outcome 